\title{
Computational Investigation on Fully Developed Periodic Laminar Flow Structure in Baffled Circular Tube with Various BR
}

\author{
Withada Jedsadaratanachai, Nuthvipa Jayranaiwachira, and Pongjet Promvonge \\ Department of Mechanical Engineering, Faculty of Engineering, King Mongkut's Institute of Technology Ladkrabang, \\ Bangkok 10520, Thailand \\ Correspondence should be addressed to Withada Jedsadaratanachai; kjwithad@kmitl.ac.th
}

Received 12 July 2013; Accepted 29 December 2013; Published 5 March 2014

Academic Editor: Tony Sheu Wen Hann

Copyright (C) 2014 Withada Jedsadaratanachai et al. This is an open access article distributed under the Creative Commons Attribution License, which permits unrestricted use, distribution, and reproduction in any medium, provided the original work is properly cited.

\begin{abstract}
This paper presents a 3D numerical analysis of fully developed periodic laminar flow in a circular tube fitted with $45^{\circ}$ inclined baffles with inline arrangement. The computations are based on a finite volume method, and the SIMPLE algorithm has been implemented. The characteristics of fluid flow are presented for Reynolds number, $\operatorname{Re}=100-1000$, based on the hydraulic diameter $(D)$ of the tube. The angled baffles were repeatedly inserted at the middle of the test tube with inline arrangement to generate vortex flows over the tested tube. Effects of different Reynolds numbers and blockage ratios $(b / D, \mathrm{BR})$ with a single pitch ratio of 1 on flow structure in the tested tube were emphasized. The flows in baffled tube show periodic flow at $x / D \approx 2-3$, and become a fully developed periodic flow profiles at $x / D \approx 6-7$, depending on Re, BR and transverse plane positions. The computational results reveal that the higher of BR and closer position of turbulators, the faster of fully developed periodic flow profiles.
\end{abstract}

\section{Introduction}

The studies of fluid flow structure and heat transfer behavior are among the most important in the design of heat exchangers in applied engineering works. Many works studied flow structure and heat transfer by using turbulators, ribs fins grooves, or baffles. It is a matter of concern that the enhancement of heat transfer rate depends on effect of turbulators that can change flow structure and behavior in the tested section.

Sripattanapipat and Promvonge [1] numerically studied the laminar periodic flow and thermal behaviors in a twodimensional channel fitted with staggered diamond-shaped baffles. They found that the diamond baffle with half apex angle of $5-10^{\circ}$ slightly performs better flow than flat baffle. Promvonge et al. [2] also examined numerically the laminar heat transfer in a square channel with $45^{\circ}$ angled baffle placed on one wall. They reported that a single streamwise vortex flow appears and also induces impingement jets on the wall of the interbaffle cavity and the BTE sidewall. Moreover, Promvonge et al. $[3,4]$ also applied the numerical manner to investigate the laminar flow structure and thermal behaviors in a square channel with $30^{\circ}$ or $45^{\circ}$ inline baffles on two opposite walls. Two streamwise counterrotating vortex flows were created along the channel lead to increased heat transfer rate over the smooth channel while the maximum thermal enhancement factors of about 2.6 at $\mathrm{BR}=0.20, \mathrm{PR}=1$, and $\mathrm{Re}=1000$ and of around 4.0 at $\mathrm{BR}=0.15, \mathrm{PR}=2$, and $\operatorname{Re}=2000$ for using the $45^{\circ}$ and $30^{\circ}$ baffles were reported, respectively.

Li and Braun [5] compared flow configuration and transport mechanisms in rectangular and cylindrical enclosures with and without baffles. They found that the presence of the baffle interrupted direct wall layer interactions and circumferential partitioning of the flow. Therefore, the transport of mass and energy was reduced in comparison to the case with no baffle. A numerical investigation of laminar forced convection in a three-dimensional channel with baffles for periodically fully developed flow and with a uniform heat flux in the top and bottom walls was conducted by Lopez et al. [6]. Guo and Anand [7] studied the three-dimensional 


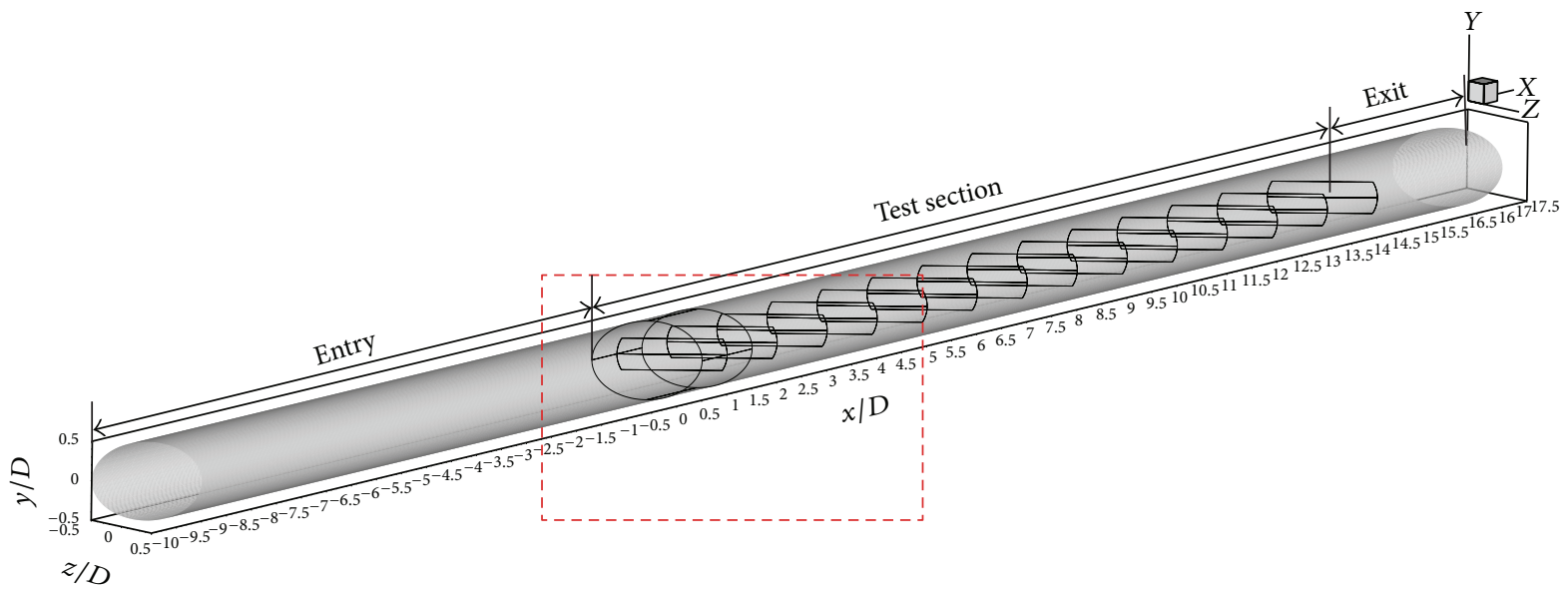

(a)

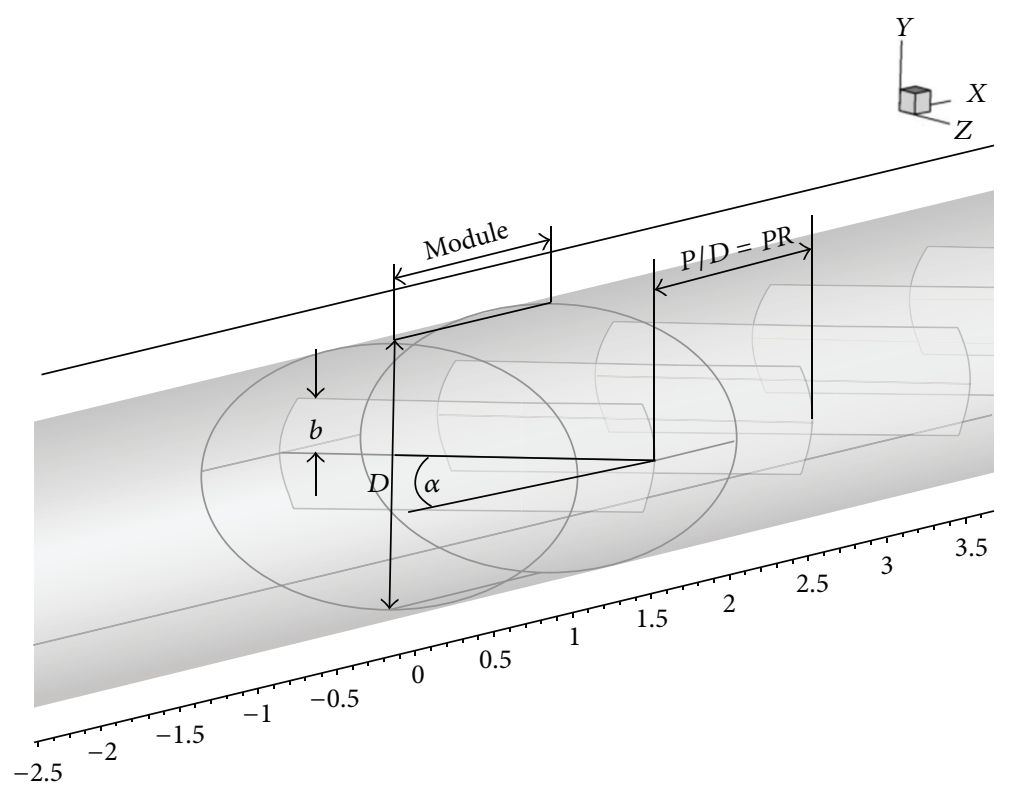

(b)

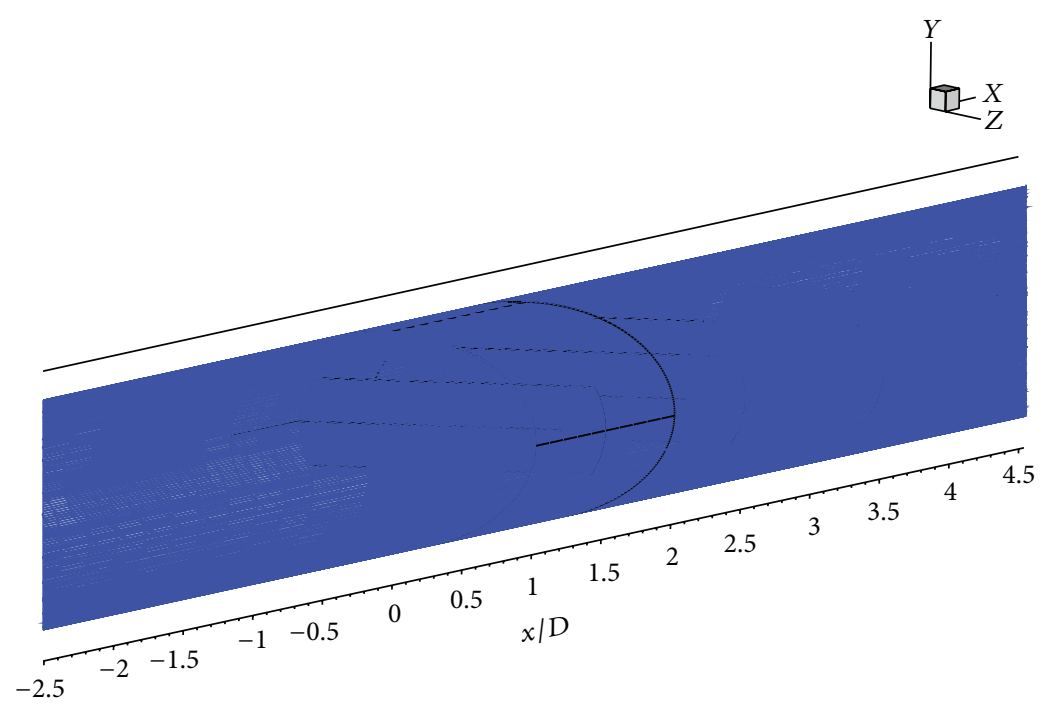

(c)

FigURE 1: (a) Circular tube geometry with $45^{\circ}$ inclined baffle inserted (b) baffled tube details, and (c) computational domain. 
heat transfer in a channel with a single baffle in the entrance region. Numerical studies for both solid and porous baffles in a two-dimensional channel for the turbulent flow [8] and for the laminar flow regimes $[9,10]$ were conducted and similar thermal performance results for both the solid and porous cases were reported. Ko and Anand [11] carried out an experiment for turbulent channel flow with porous baffles and found that the case with porous baffles presents a flow behavior as good as the one with solid baffles. Tsay et al. [12] investigated numerically by using baffles for enhancement of heat transfer in laminar channel flow over two heated blocks mounted on the lower plate.

The concept of periodically fully developed flow was widely used. However, most investigations studied only effects of turbulators and their parameters. Therefore, the study on developing of periodic flow structure concept has rarely been reported. In this present work, the numerical computations for three-dimensional laminar periodic channel flows over a $45^{\circ}$ inclined baffle are conducted to examine the changes in the flow structure and a way to develop to fully developed periodic flow profile.

\section{Flow Descriptions}

2.1. Baffle Geometry and Arrangement. The system of interest is a horizontal circular tube with inclined baffles which are repeatedly inserted at the middle of the test tube as shown in Figure 1(a). The circular tube is divided into 3 parts: entry region, test section, and exit. The details of the baffled tube and computation domain and its grid are shown in Figures 1(b) and 1(c), respectively. The air enters the tube at an inlet temperature, $T_{\text {in }}$, and flows over baffle where $b$ is the baffle height, $D$, set to $0.05 \mathrm{~m}$, is the tube diameter, and $b / D$ is known as the blockage ratio, $\mathrm{BR}=0.10-0.25$. The distance between the inclined baffle cell or axial pitch, $L$, is set to $L=D$ in which $L / D$ is defined as the pitch spacing ratio, $P R$, and set to 1 . To investigate an effect of Reynolds number, $\mathrm{Re}=100-$ 1000 .

2.2. Boundary Conditions. For baffled circular tube, a uniform air velocity is introduced at the inlet while a pressure outlet condition is applied at the exit. The physical properties of the air have been assumed to be constant at mean bulk temperature. Impermeable boundary and no-slip wall conditions have been implemented over the tube walls as well as the baffle.

\section{Mathematical Foundation}

The numerical model for fluid flow in a circular tube was developed under the following assumptions.

(i) Steady, three-dimensional, laminar, and incompressible fluid flows.

(ii) Constant fluid properties.

(iii) Ignoring body forces.

Based on the above assumptions, the tube flow is governed by the continuity and the Navier-Stokes equations.

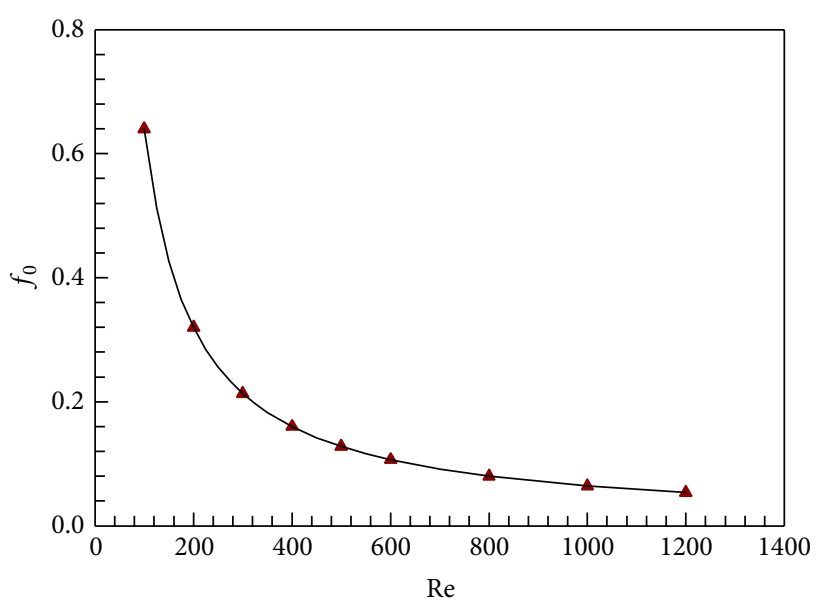

- Present prediction

- $f \operatorname{Re}=64$, tube

FIgURE 2: Verification of friction factor for smooth circular tube with no baffle.

In the Cartesian tensor system, these equations can be written as follows.

Continuity equation:

$$
\frac{\partial}{\partial x_{i}}\left(\rho u_{i}\right)=0
$$

Momentum equation:

$$
\frac{\partial\left(\rho u_{i} u_{j}\right)}{\partial x_{j}}=-\frac{\partial p}{\partial x_{i}}+\frac{\partial}{\partial x_{j}}\left[\mu\left(\frac{\partial u_{i}}{\partial x_{j}}+\frac{\partial u_{j}}{\partial x_{i}}\right)\right]
$$

Apart from the energy equation discretized by the QUICK scheme, the governing equations were discretized by the power law scheme, decoupling with the SIMPLE algorithm and solved using a finite volume approach [13]. The solutions were considered to be converged when the normalized residual values were less than $10^{-5}$ for all variables.

The Reynolds number is defined as

$$
\operatorname{Re}=\frac{\rho \bar{u} D}{\mu} .
$$

The friction factor, $f$, is computed by pressure drop, $\Delta p$, across the length of the periodic channel, $L$, as

$$
f=\frac{(\Delta p / L) D}{(1 / 2) \rho \bar{u}^{2}}
$$

The variation in $f$ values for the $45^{\circ}$ inclined baffle at $\mathrm{BR}=0.20$ and $\mathrm{Re}=600$ is less than $0.2 \%$ with increasing the number of cells from 500,000 to 800,000 . Therefore, there is no such advantage in increasing the number of cells beyond this value and, thus, the grid system of 500,000 cells is adopted for the current computation. 


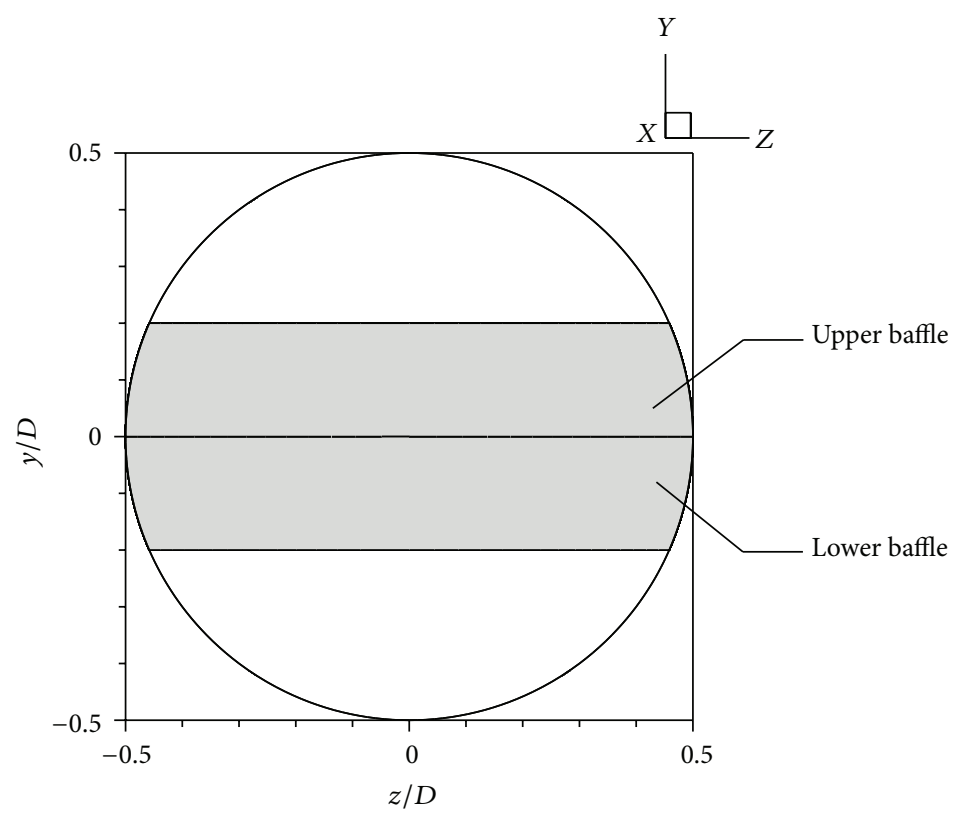

Figure 3: $Y Z$ sxis of baffled tube.

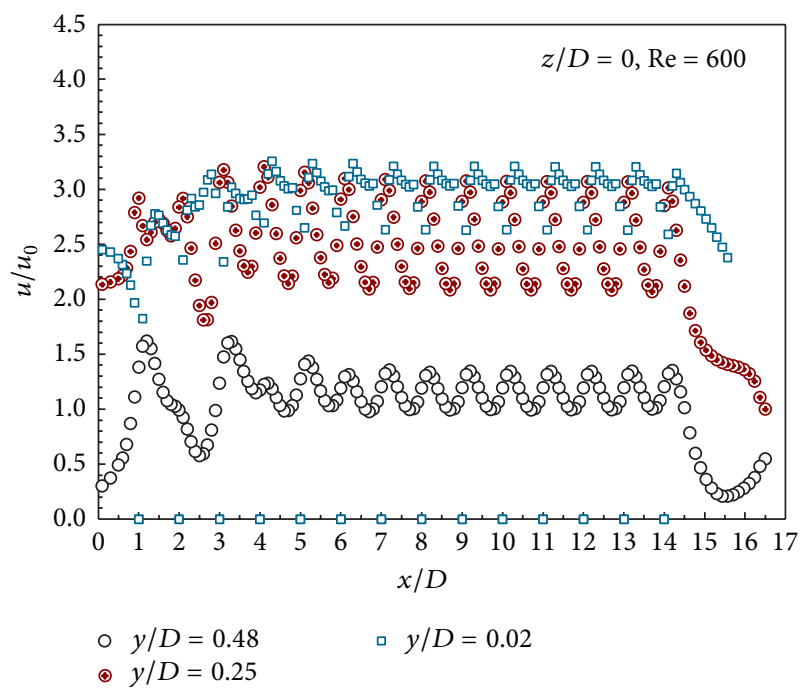

Figure 4: Velocity profile at $z / D=0$ with various $y / D$ value for $\mathrm{BR}=0.20$ and $\mathrm{Re}=600$.

\section{Results and Discussion}

The results of $45^{\circ}$ inclined baffle with inline arrangement in circular tested tube are expressed in six parts, such as validation, fully developed periodic concept, effect of transverse plane position, flow structure, effect of Reynolds number, and effect of blockage ratio.

4.1. Validation. Verification for the friction factor of the smooth circular tube with no baffle is initially performed by a comparison with the values from previous correlations under a similar operating condition as shown in Figure 2. The present numerical smooth duct result shows the excellent

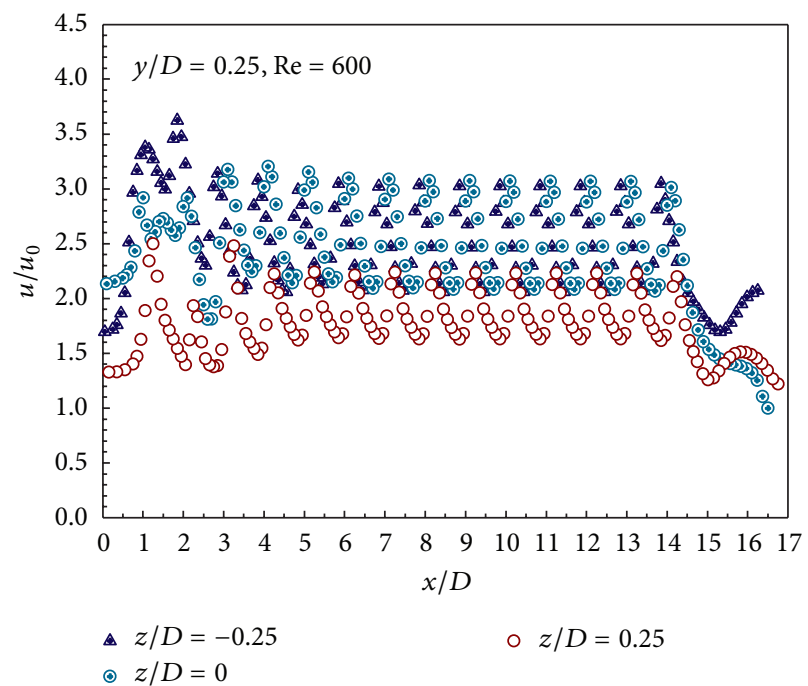

FIGURE 5: Velocity profile at $y / D=0.25$ with various $z / D$ value for $\mathrm{BR}=0.20$ and $\mathrm{Re}=600$.

agreement with correlation solutions obtaining from the open literature [14] for friction factor, within $\pm 1.5 \%$ maximum deviations.

4.2. Fully Developed Periodic Concept. Understanding of a fully developed periodic profile condition in the baffled tube is needed before discussing the results. The fully developed periodic flow conditions in the tube mounted repeatedly with inline $45^{\circ}$ inclined baffles can be displayed by considering the axial $u / u_{0}$ distribution as depicted in Figures 4 and 5 . The $u / u_{0}$ distributions of a baffled test tube are presented at different transverse plane positions as Figure 3. 


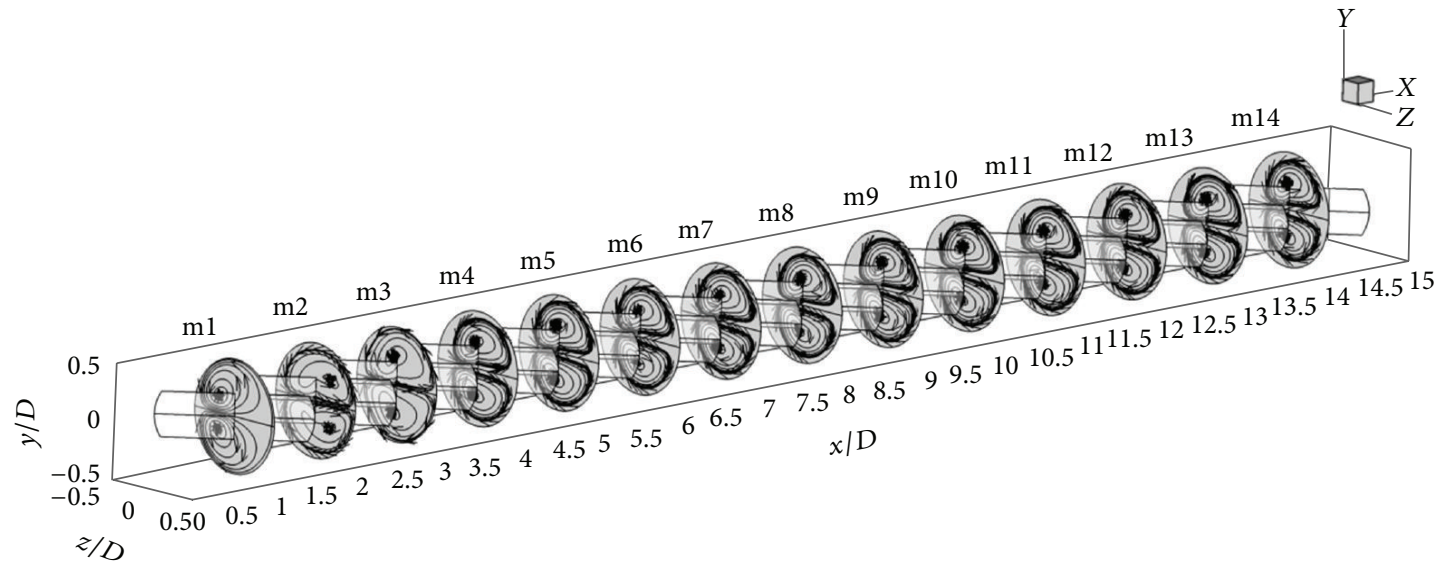

FIGURE 6: Streamline in transverse plane for $\mathrm{BR}=0.20$ at $\mathrm{Re}=600$.

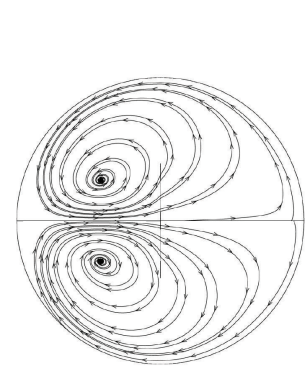

$\mathrm{m} 1$

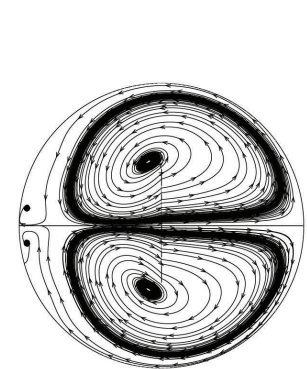

$\mathrm{m} 5$

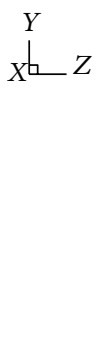

$\mathrm{m} 2$
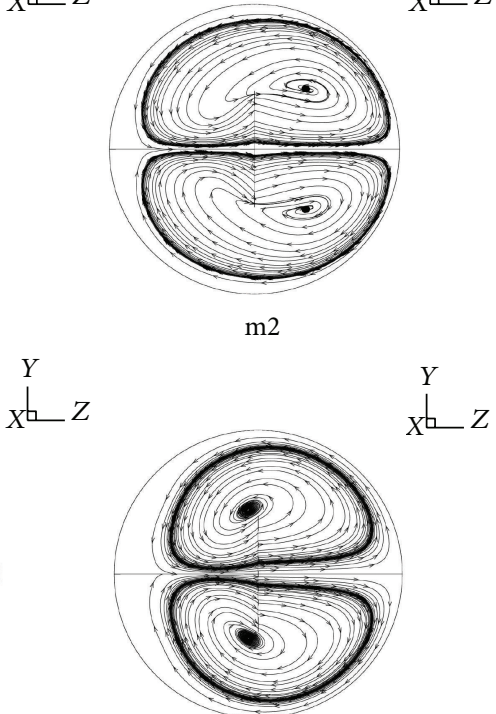

m6

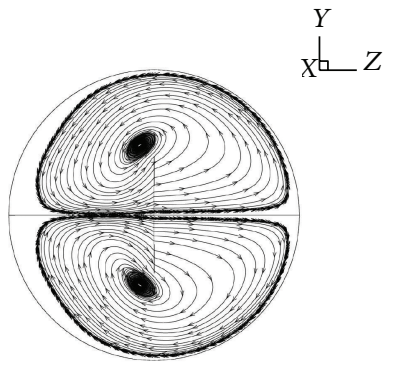

$\mathrm{m} 3$

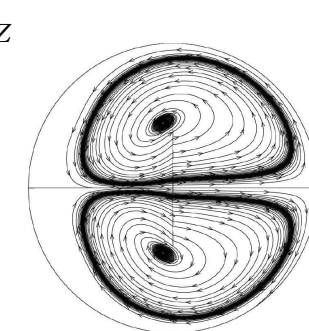

$\mathrm{m} 7$

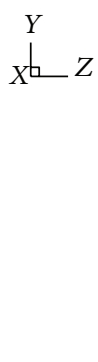

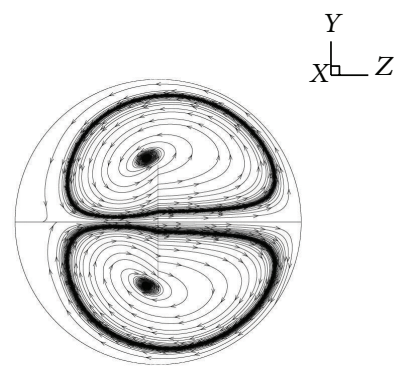

$\mathrm{m} 4$

Y

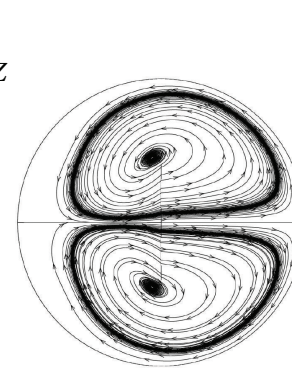

$\mathrm{m} 8$

FIGURE 7: Streamline in transverse planes $\mathrm{m} 1$ to $\mathrm{m} 8$ for $\mathrm{BR}=0.20$ at $\mathrm{Re}=600$.

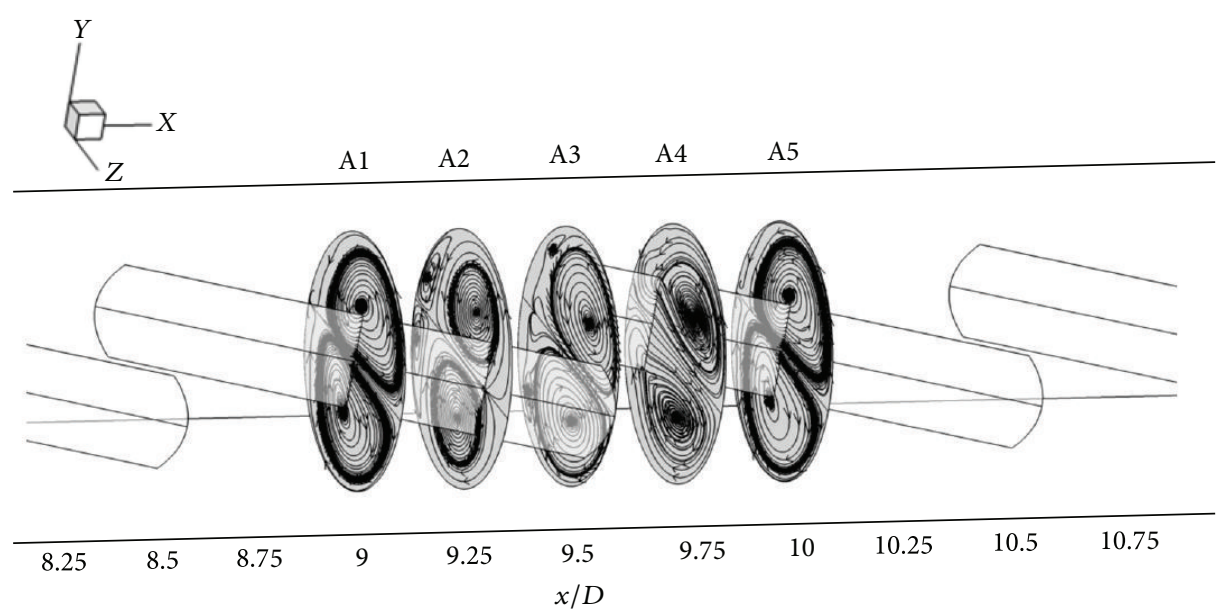

FIgURE 8: Streamline in transverse plane for a module at $\mathrm{BR}=0.20$ at $\mathrm{Re}=600$. 

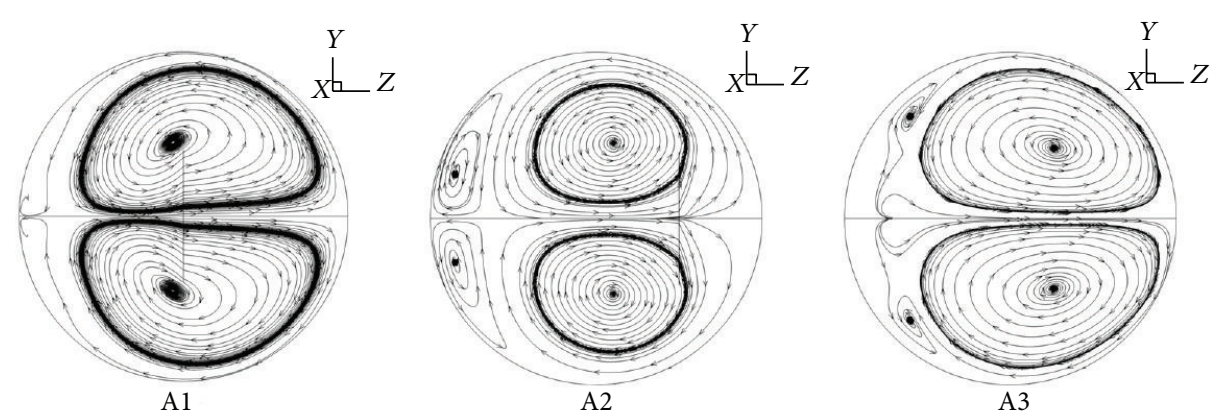

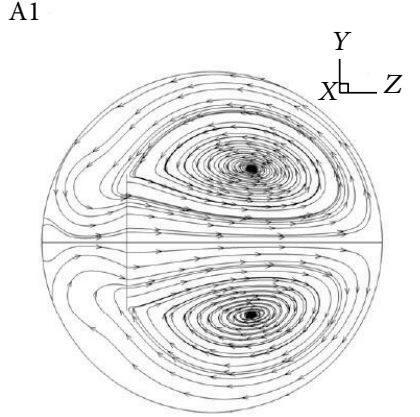

A4

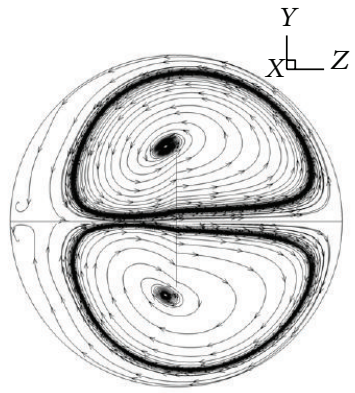

A 5

FIgURE 9: Streamline in transverse planes A1 to A5 for a module at $\mathrm{BR}=0.20$ at $\mathrm{Re}=600$.

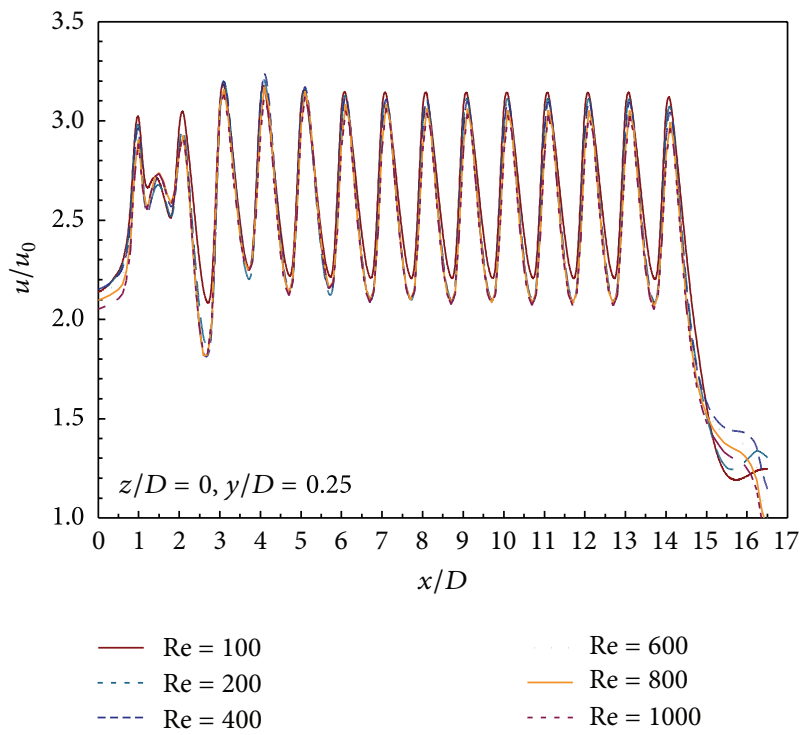

FIGURE 10: Velocity profile at $z / D=0$ and $y / D=0.25$ with various Re values for $\mathrm{BR}=0.20$.

The profile of velocity is explained into 2 forms. First, the velocity profile that structures similarity to other modules but the values are not equal as shown in Figures 4 and 5 at $x / D=$ $3-4$ and $x / D=2-3$, respectively; this profile is called "periodic flow profile". Second, the profile that equal on both structures and values is called "fully developed periodic flow profile" as Figures 4 and 5 when the $x / D \geq 7$.

4.3. Effect of Transverse Plane Position. The effect of transverse plane position is expressed into 2 axes, such as $y / D$ and $z / D$, as seen in Figures 4 and 5, respectively.
Figure 4 displays the axial $u / u_{0}$ profiles along the baffled tube with $\mathrm{BR}=0.2$ and $\mathrm{Re}=600$ at location $z / D=0$ with different $y / D$ values of $0.02,0.25$ and 0.48 . It can be seen in the figure that the $u / u_{0}$ profiles for all cases become periodic at the 2 nd module and tend to increase to a fully developed periodic flow at about the 6th-7th module or at $x / D \approx 6-7$. In addition, the $u / u_{0}$ profile at the location $y / D=0.02$ close to baffle becomes a fully developed periodic flow profile slightly faster than that at $y / D=0.25$ and 0.48 .

Figure 5 shows the axial distribution of $u / u_{0}$ for $y / D=0.25$ with $z / D$ values of $-0.25,0$ and 0.25 . In this figure, it is apparent that the $u / u_{0}$ profiles for different $z / D$ locations at a fixed location of $y / D=0.25$ have no effect for fully developed periodic flow regions while those for different $y / D$ locations at a fixed point of $z / D=0.25$ show different locations to obtain the fully developed periodic flow regime. In closer inspection, the flow in the region closer to the baffle becomes a fully developed periodic profile faster than that farther away from the baffle.

Therefore, the concept of fully developed periodic flow profile can be applied efficiently to laminar tube flow through baffles if the test tube is sufficiently long $(x / D>10$ is preferable, in general). It can be observed that the mean $u / u_{0}$ value is not much different for both the periodic regions. Further, with considering both convergent time and solution precision, only a fully developed periodic flow tube model is employed in the computation.

4.4. Flow Structure. The flow structure in the tube repeatedly mounted with the inline $45^{\circ}$ inclined baffles can be displayed by considering the streamline in transverse planes for $\mathrm{Re}=$ 600 and $\mathrm{BR}=0.20$ as shown in Figures 6-9. By partially 


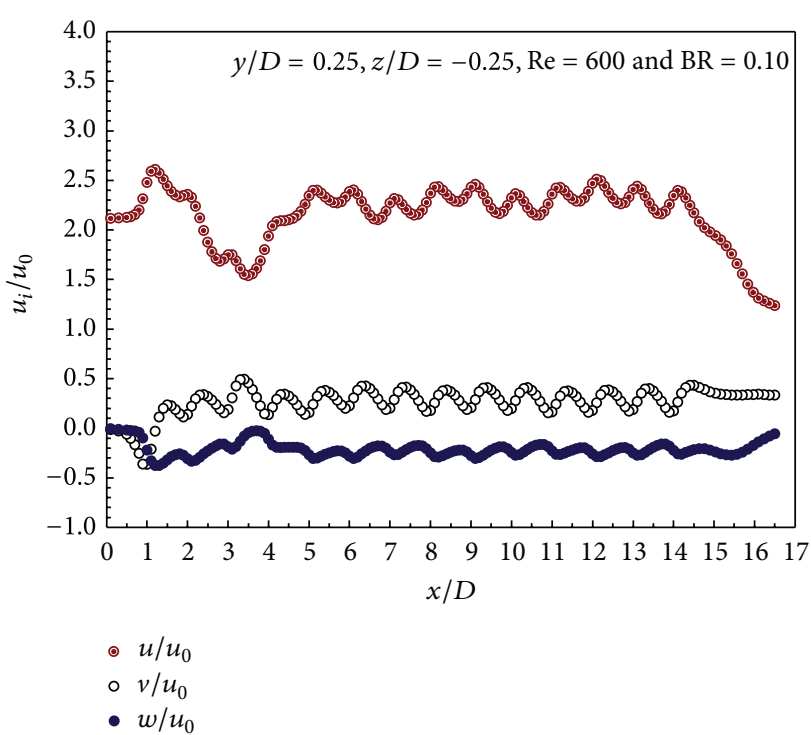

(a)

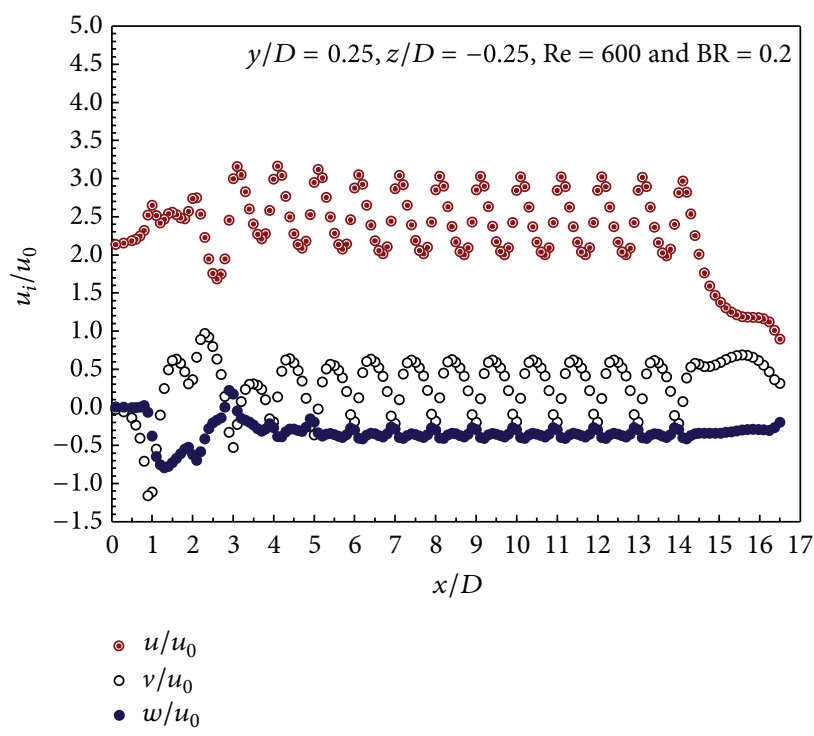

(c)

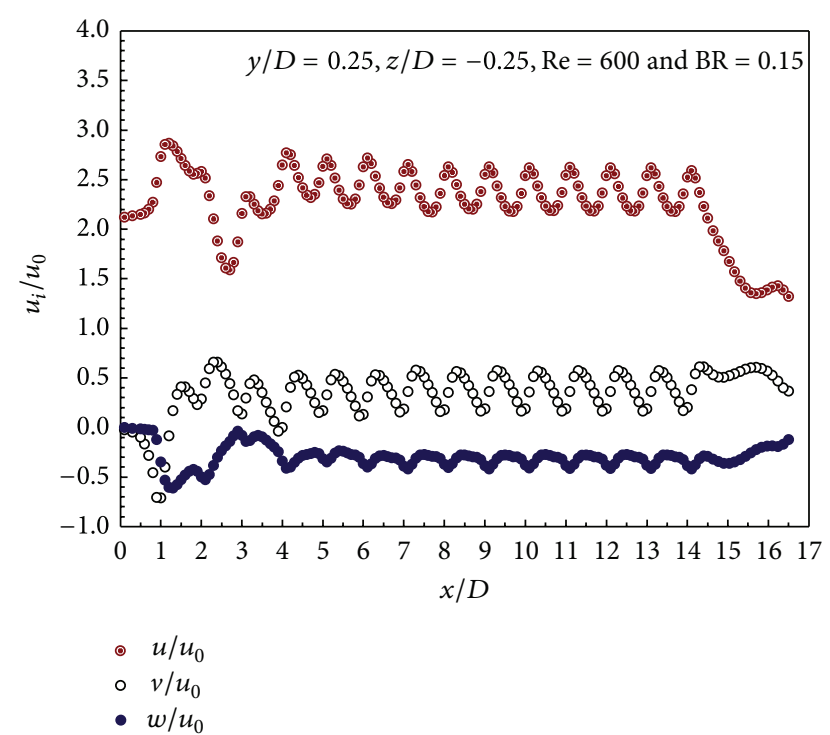

(b)

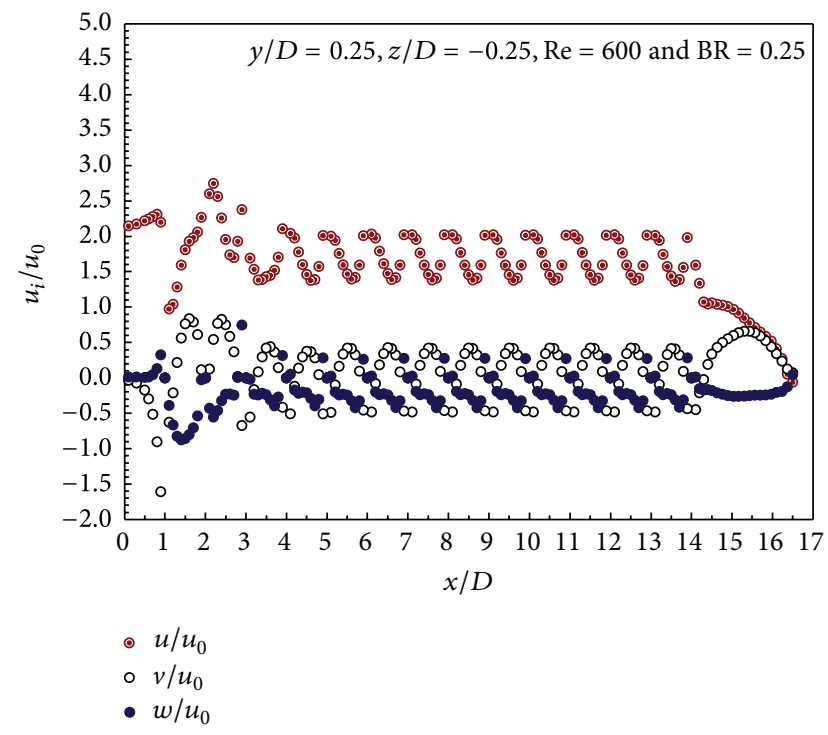

(d)

FIGURE 11: Velocity profile at $y / D=0.25, z / D=-0.25$ and $\mathrm{Re}=600$ for (a) $\mathrm{BR}=0.10$, (b) $\mathrm{BR}=0.15$, (c) $\mathrm{BR}=0.20$, and $(\mathrm{d}) \mathrm{BR}=0.25$.

describing the flow structure into 2 parts, the developing flow structure and the fully developed periodic flow profile.

The concept of fully developed periodic flow profile is shown in Figures 6 and 7 for overall streamline planes and the details of planes $\mathrm{m} 1$ to $\mathrm{m} 8$, respectively. Streamline in transverse planes of each module is plotted at a similar position, in the middle of every element. In Figure 6 the flow profiles are found to be similar as planes $\mathrm{m} 4$ to $\mathrm{m} 14$. The detail of streamline in transverse planes is shown in Figure 7 for only $\mathrm{m} 1$ to $\mathrm{m} 8$; causes $\mathrm{m} 9$ to $\mathrm{m} 14$ give similar structure as depicted in Figure 6. It is visible that the plane mland the two counterrotating vortex pairs caused by the baffle appear on the lower and upper parts of the module. The core of pair vortex appears at the left of the plane from the middle of plane $(z / D=0)$. The next module of plane $\mathrm{m} 2$ and the two counterrotating vortex appears similarly to $\mathrm{ml}$ but the position of vortex core shows a difference as seen at the right of the plane. Planes $\mathrm{m} 3$ to $\mathrm{m} 8$ show similarity on both flow and vortex core position, at the middle of the plane and on the baffle position. In addition, the result from the streamline in the transverse plane plotted can be describing flow structure and periodic flow profile but the fully developed periodic flow profile must be explained by the values of velocity.

The change of flow structure in fully developed periodic flow profile (Figure 8) from the start to the end of module at $x / D=9-10$ is presented and the details of each plane, $\mathrm{A} 1$ to A5, are shown in Figure 9. Plane A1, at the middle of the baffle point, two counterrotating vortexes from left to right, and vortex core appear in the middle of the plane over 
the baffle; after that, the vortex core moves along the baffle to plane A2 and appear the new pairs of vortex on the left of the plane. Plane A3 is the end of baffle at the right plane and start point of baffle at the left plane; vortex core before gradually faded and the new one will be appear. Plane A4, the new core of vortex, moves along the baffle as well as next plane, A5. The flow structure presents repeatedly from plane A1 to A5 in the fully developed periodic flow profile region.

4.5. Effect of Re. The effect or Re is described by considering $u / u_{0}$ profile versus $x / D$ at different Re as depicted in Figure 10; the inclined baffles with $\mathrm{BR}=0.20$ at $z / D=0$ and $y / D=0.25$ for Re in range from 100 to 1000 are presented. In this figure, the flow structure along the $x$-axis gives similarity structure for all Re values. The flow characteristic begins periodic flow at $x / D \approx 2-3$ and becomes a fully developed periodic flow profile at $x / D \approx 6-7$ for all cases. This means that the Re is not effective for the developing of fully developed periodic flow structure.

4.6. Effect of BR. The effect of $\mathrm{BR}$ can be displayed by considering the velocity profile, $u_{i} / u_{0}$, as depicted in Figures $11(\mathrm{a}), 11(\mathrm{~b}), 11(\mathrm{c})$, and $11(\mathrm{~d})$ for $\mathrm{BR}=0.10,0.15,0.20$, and 0.25 at $\operatorname{Re}=600, y / D=0.25$, and $z / D=-0.25$, respectively.

In Figure 11(a), the $v / u_{0}$ profile shows periodic profile at $x / D \approx 2-3$ and becomes fully developed periodic flow profile at $x / D \approx 9-10$. The $u / u_{0}$ profile provides periodic profile at $x / D \approx 5-6$ but does not become fully developed periodic flow structure. The $w / u_{0}$ profile shows periodic profile and fully developed periodic flow profile at $x / D \approx 2-3$ and $x / D \approx 11-$ 12 , respectively. In addition, the flow structure of $\mathrm{BR}=0.10$ does not seem to be going to be fully developed periodic flow profile in range of 14 modules tested. Fully developed periodic flow concept for $\mathrm{BR} \leq 0.10$ will appear when increasing the module of test section or decreasing the distance between baffle, $P R<1$.

In Figures 11(b), 11(c), and 11(d), BR = 0.15-0.25; $u / u_{0}$ profile presents periodic at $x / D \approx 2-3$ and becomes fully developed periodic at $x / D \approx 6-7$ when $v / u_{0}$ and $w / u_{0}$ develop into fully developed periodic flow profile slightly faster than $x$-velocity around a half to one module. This can be attributed that the fully developed periodic flow profiles perform faster when increasing BR.

\section{Conclusions}

3D laminar fully developed periodic flow characteristics in a circular test tube mounted repeatedly with inline $45^{\circ}$ inclined baffles inserted in the middle of the test tube for 14 modules are numerically investigated.

The baffled tube flow shows periodic flow profile at $x / D \approx 2-3$ and becomes fully developed periodic flow profiles at $x / D \approx 6-7$ downstream of the tested tube inlet for $\mathrm{BR}=0.15-0.25, \mathrm{PR}=1$, and $\mathrm{Re}=100-1000$ when $\mathrm{BR}=0.10$ does not become fully developed periodic flow. The fully developed periodic flow profile concept depends on BR and transverse plane position. the increasing BR and close to the baffle regimes are the factors for occurring the fully developed periodic flow profile. The Re in range of study has no effect for fully developed periodic flow profile.

Thus, the fully developed periodic flow structure and behavior concept in tested tube at $\mathrm{BR} \geq 0.15, \mathrm{PR}=1$, and $\mathrm{Re}=100-1000$ are recommended for use instead of the full tube and all of systems in order to save more resources and time in numerical investigation. In case of $\mathrm{BR} \leq 0.10$, the fully developed periodic flow profile concept may be used for PR $<1$ and the tested section is higher than 14 modules.

\section{Conflict of Interests}

The authors declare that there is no conflict of interests regarding the publication of this paper.

\section{Acknowledgment}

The authors would like to gratefully thank the King Mongkut's Institute of Technology Ladkrabang (KMITL) for the financial support of the research.

\section{References}

[1] S. Sripattanapipat and P. Promvonge, "Numerical analysis of laminar heat transfer in a channel with diamond-shaped baffles," International Communications in Heat and Mass Transfer, vol. 36, no. 1, pp. 32-38, 2009.

[2] P. Promvonge, S. Sripattanapipat, S. Tamna, S. Kwankaomeng, and C. Thianpong, "Numerical investigation of laminar heat transfer in a square channel with $45 \circ$ inclined baffles," International Communications in Heat and Mass Transfer, vol. 37, no. 2, pp. 170-177, 2010.

[3] P. Promvonge, S. Sripattanapipat, and S. Kwankaomeng, "Laminar periodic flow and heat transfer in square channel with $45^{\circ}$ inline baffles on two opposite walls," International Journal of Thermal Sciences, vol. 49, no. 6, pp. 963-975, 2010.

[4] P. Promvonge, W. Jedsadaratanachai, and S. Kwankaomeng, "Numerical study of laminar flow and heat transfer in square channel with $30^{\circ}$ inline angled baffle turbulators," Applied Thermal Engineering, vol. 30, no. 11-12, pp. 1292-1303, 2010.

[5] H. Li and M. J. Braun, "Flow structure and transport mechanism in lower half heated upper half cooled enclosures in laminar flow regime," Heat and Mass Transfer, vol. 42, no. 9, pp. 823834, 2006.

[6] J. R. Lopez, N. K. Anand, and L. S. Fletcher, "Heat transfer in a three-dimensional channel with baffles," Numerical Heat Transfer A, vol. 30, no. 2, pp. 189-205, 1996.

[7] Z. Guo and N. K. Anand, "Three-dimensional heat transfer in a channel with a baffle in the entrance region," Numerical Heat Transfer A, vol. 31, no. 1, pp. 21-35, 1997.

[8] Y.-T. Yang and C.-Z. Hwang, "Calculation of turbulent flow and heat transfer in a porous-baffled channel," International Journal of Heat and Mass Transfer, vol. 46, no. 5, pp. 771-780, 2003.

[9] B. M. da Silva Miranda and N. K. Anand, "Convective heat transfer in a channel with porous baffles," Numerical Heat Transfer A, vol. 46, no. 5, pp. 425-452, 2004.

[10] N. B. Santos and M. J. S. de Lemos, "Flow and heat transfer in a parallel-plate channel with porous and solid baffles," Numerical Heat Transfer A, vol. 49, no. 5, pp. 471-494, 2006. 
[11] K.-H. Ko and N. K. Anand, "Use of porous baffles to enhance heat transfer in a rectangular channel," International Journal of Heat and Mass Transfer, vol. 46, no. 22, pp. 4191-4199, 2003.

[12] Y.-L. Tsay, J.-C. Cheng, and T.-S. Chang, "Enhancement of heat transfer from surface-mounted block heat sources in a duct with baffles," Numerical Heat Transfer A, vol. 43, no. 8, pp. 827-841, 2003.

[13] S. V. Patankar, "Numerical heat transfer and fluid flow," 1980.

[14] F. Incropera and P. D. Dewitt, Introduction to Heat Transfer, John Wiley \& Sons, 5th edition, 2006. 


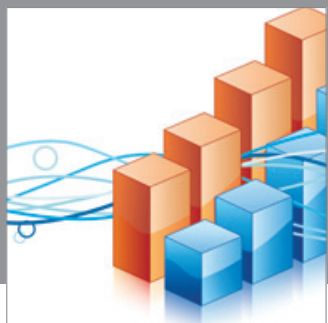

Advances in

Operations Research

mansans

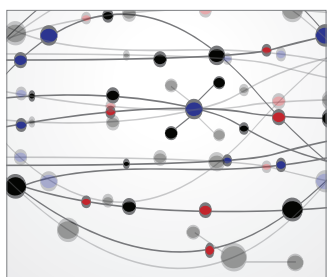

The Scientific World Journal
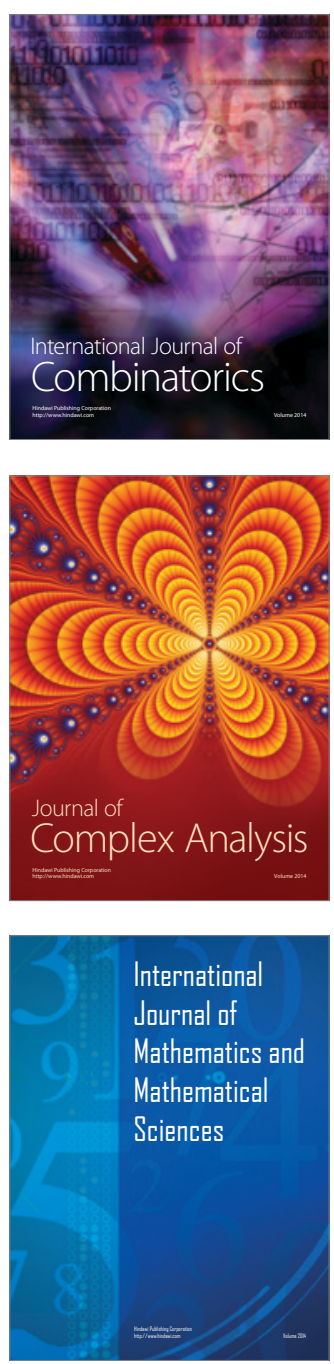
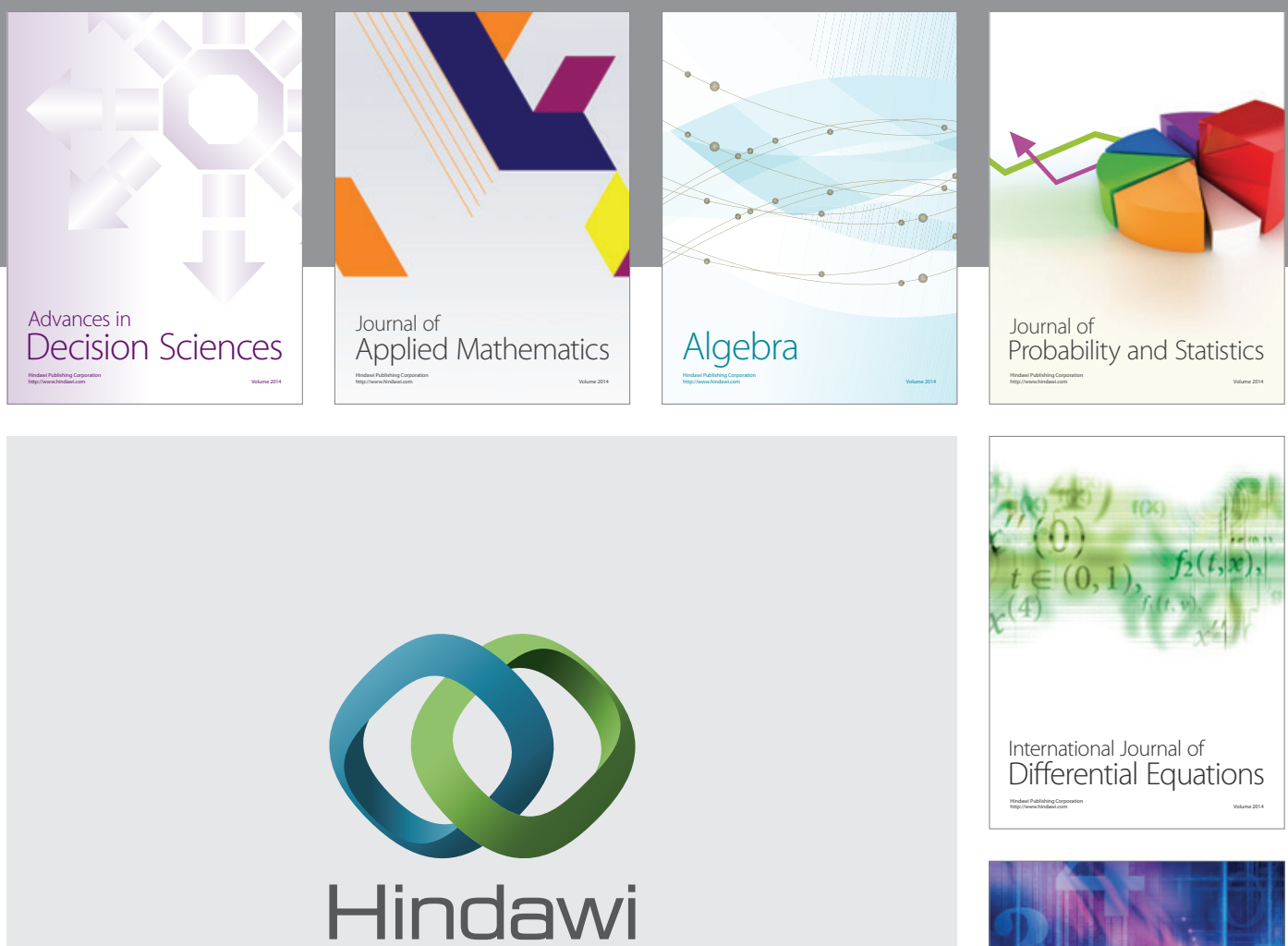

Submit your manuscripts at http://www.hindawi.com
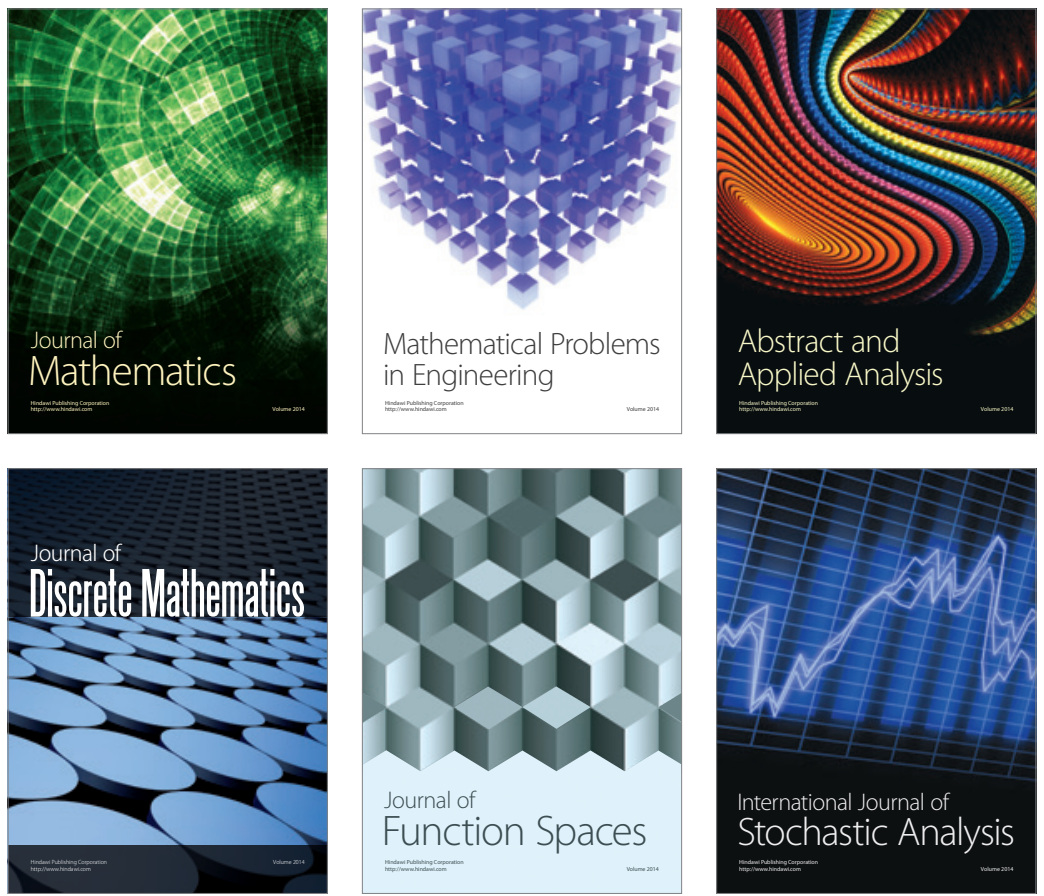

Journal of

Function Spaces

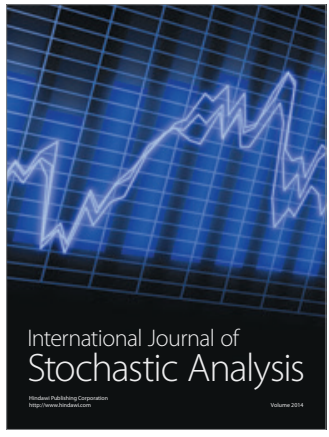

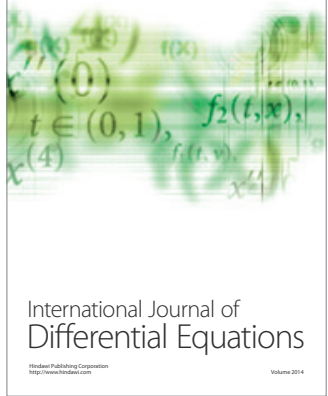
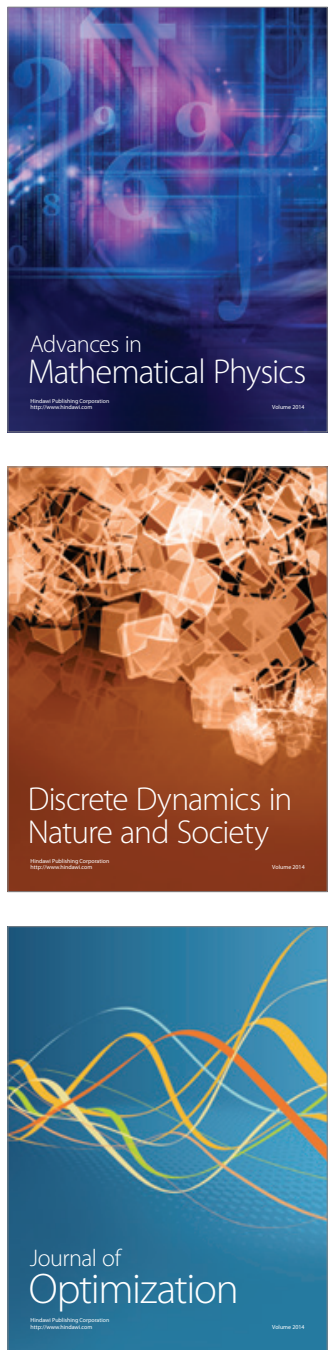